\title{
Vibrations of water hydraulic systems - an experimental approach
}

\author{
Andrei Dragomirescu ${ }^{1}$, Carmen-Anca Safta $^{1,}{ }^{*}$, Nicolae Orăşanu ${ }^{2}$, Ioan Magheți ${ }^{2}$, and Lucian Mândrea ${ }^{1}$ \\ ${ }^{1}$ Univ. Politehnica of Bucharest, Faculty of Power Eng., 313 Splaiul Independentei, 060042, Bucharest, Romania \\ ${ }^{2}$ Univ. Politehnica of Bucharest, Dept. of Mechanics, 313 Splaiul Independentei, 060042, Bucharest, Romania
}

\begin{abstract}
This paper presents an experimental study of the vibrations induced both by cavitating and by non-cavitating flow in a hydraulic installation that comprises the main elements of a water hydraulic system. The cavitation was triggered by progressively closing a butterfly valve. The vibrations were measured on the pump, on the bearings housing, on the pump drive motor, and at nine measurement points located upstream and downstream of the main elements of the installation. The measurements were carried out at different flow rates obtained at different openings of the butterfly valve. The results suggest that the phenomena that take place inside the pump and inside the bearings cause vibrations having frequencies of up to $10 \mathrm{kHz}$. The results also indicate that the cavitation that occurs at the butterfly valve causes vibrations of high frequency, above $3 \mathrm{kHz}$, that have a distinct peak at about $18 \mathrm{kHz}$. These results could be useful in establishing proper maintenance plans for hydraulic installations.
\end{abstract}

\section{Introduction}

The current state of the fundamental and industrial research regarding vibrations in water hydraulic systems reveals that the vibrations are the effect of the operating conditions of valves and pumps [1-5]. Pumps operating at partial-flow regime are a frequent cause of vibrations (pump-induced vibrations) in water piping systems [6].

Some vibrations are the effect of cavitation phenomena which occur in the flow section of throttling devices (valves, orifices, nozzles) when the pressure drops below the vapor pressure of the fluid at the process temperature. Once the cavitation begins, critical operating conditions could occur, that have different effects, such as increased noise emissions, pressure fluctuations and vibrations, erosion, corrosion, and loss in installation efficiency. Eventually, structural failure of a pipe hydraulic system could occur because of fatigue caused by supercavitation that is accompanied by maximum noise and vibration levels $[4,7]$. The vibration of the entire hydraulic system is a measure of the system integrity and the vibration measurements "quantify" the effects of the transient movements of flow instabilities and cavitation.

The experimental studies presented in the literature show the characteristic frequency bands specific to cavitation-induced vibrations for different types of devices found in water hydraulic systems. For a butterfly valve, frequencies of $4 \mathrm{kHz}$ and $9.6 \mathrm{kHz}$ were identified to be specific to cavitation [8]. In the case of a hollow jet valve, the frequency band of the cavitation induced vibrations is between $5 \mathrm{kHz}$ and $6 \mathrm{kHz}$, and another band is between $10 \mathrm{kHz}$ and $23 \mathrm{kHz}$ [9].
The frequency analysis of cavitation-induced vibrations highlights a high-frequency vibration domain that can be monitored at each level of cavitation intensity. It was experimentally observed that, in advanced stages of cavitation, the cavitation number $\sigma$ decreases to lower values (e.g. $\sigma<1$ in the case of supercavitation) and the vibration amplitudes increase to values higher than $1 \mathrm{~m} / \mathrm{s}^{2}$ $[4,10]$.

In water hydraulic systems, the flow-induced vibrations are represented in the low-frequency band as well. One cause of low-frequency vibrations is flashing. Downstream of the narrow section where cavitation normally occurs, flashing begins, replacing cavitation, when the pressure difference between the downstream pressure and the vapor pressure becomes less than or equal to zero. In such a case, the vapor bubbles do not implode (collapse) anymore and a two-phase flow (a liquid-vapor mixture) develops [11, 12]. Although flashing is not as severe as cavitation, it cannot be controlled or prevented [13]. The effects of flashing flow are flashing erosion and low-frequency vibrations. When the flashing flow appears in an installation with narrow sections, the entire pipeline responds and becomes excited to mechanical vibrations having frequencies below $10 \mathrm{~Hz}[11]$.

The vibroacoustic behavior of hydraulic installations depends on the physical properties of the fluid inside, on the pipe material and geometry (such as cross-section, wall thickness, etc.), and on each component of the hydraulic system (pipes, valves, pumps, flanges, elbows with welding joints, and other pipe connections) [5].

This paper presents results of the vibration measurements on a water hydraulic installation at different operating conditions. The measurements were carried out

\footnotetext{
*Corresponding author: safta.carmenanca@gmail.com
} 
at twelve measurement locations, including the pump, the bearings housing, and the drive motor. Three accelerometers were used to record the vibrations. The acquired signals are analyzed and discussed based on Fast Fourier Transforms (FFTs) and Root Mean Square (RMS) accelerations. A synoptic diagram with the behavior of the analyzed water hydraulic system is presented. Of a particular interest was to identify the frequency domains of the vibrations caused by the cavitation at the butterfly valve.

\section{Experimental installation measurement procedure}

and

The experiments were carried out at a water hydraulic installation which was designed as a test rig for butterfly valves having the nominal diameter of $100 \mathrm{~mm}$ and the nominal pressure of 6 bar (Dn 100, Pn 6 [7]).

The installation (Fig. 1) is an open loop system that contains a free surface water tank, a suction pipe, a pumping assembly, and a discharge pipe. Both the suction and the pressure pipe are connected to the water tank. The two pipes have the inner diameters $D_{1}=128 \mathrm{~mm}$ and $D_{2}=104 \mathrm{~mm}$ respectively.

An orifice plate with $D$ and $D / 2$ pressure tappings is mounted on the suction pipe for measuring the flow rate. The differential pressure at the orifice plate is measured with an U-tube differential manometer with mercury.

The pumping assembly consists in a centrifugal pump driven by a three-phase AC motor with 2 pole pairs and a rated speed of $1460 \mathrm{rpm}$. The fundamental frequency corresponding to the rated speed is of $24.3 \mathrm{~Hz}$. The pump impeller has 5 blades, hence the blade passing frequency is of roughly $121.5 \mathrm{~Hz}$. The pump shaft is supported by two ball bearings and is connected to the motor by an elastic pin bush coupling. At zero head, the pump delivers a maximum flow rate $Q_{\max }=0.043 \mathrm{~m}^{3} / \mathrm{s}$ (43 l/s).

At the pump outlet a gate valve is installed, having the role of a throttling valve that allows to correctly start and stop the pump and to adjust the flow rate independently of the butterfly valve.

The butterfly valve is of wafer type and is mounted on the pressure pipe between two flanges. Its inner surface is rubber lined to provide the seal against the valve flap. The valve is provided with a blocking mechanism that allows the adjustment of the valve angle (i.e. flap angle) between $0^{\circ}$ (fully opened) and $90^{\circ}$ (fully closed) in increments of $10^{\circ}$.

The vibration levels were measured with three

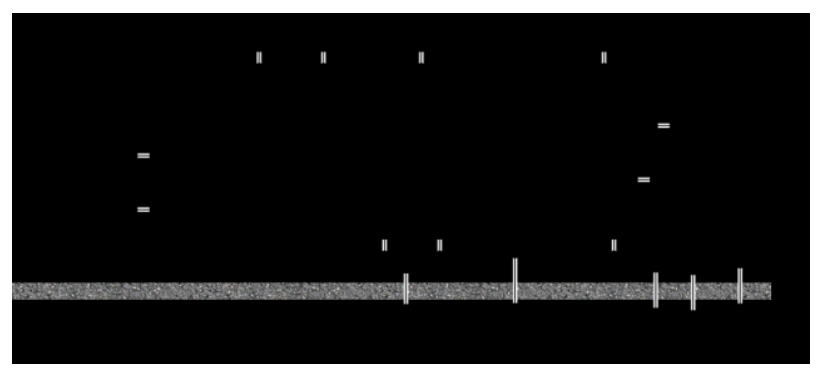

Fig. 1. Experimental installation and measurement points. identical Brüel \& Kjaer DeltaTron accelerometers with built-in preamplifier and transducer electronic data sheet (TEDS), connected to a laptop via a portable buspowered USB carrier from National Instruments. The accelerometers have the reference sensitivity of $20 \mathrm{~m} / \mathrm{s}^{2}$ RMS at $159.2 \mathrm{~Hz}$, the measuring range $\pm 7000 \mathrm{~m} / \mathrm{s}^{2}$ peak, the frequency range $10 \%$ of $0.1 \mathrm{~Hz}$ to $6 \mathrm{kHz}$, and the phase response $\pm 5^{\circ}$ of $0.5 \mathrm{~Hz}$ to $5 \mathrm{kHz}$. The sampling frequency for all the measurements was of $50 \mathrm{kHz}$. Although the sampling frequency is outside the frequency range $10 \%$, this is likely to affect only the measured amplitudes, while the relevant frequencies will still be correctly identified.

Measurements were carried out simultaneously using the three accelerometers placed at the points indicated in Figure 1. These points were grouped for the measurements as follows:

- $\quad$ points P1, P2, and P3 located downstream of the pump, on the pressure pipe;

- $\quad$ points P4 and P5 located upstream and downstream of the butterfly valve respectively, and point P6 located on the tank;

- $\quad$ points P7, P8, and P9 located on the suction pipe;

- $\quad$ points $\mathrm{P}, \mathrm{B}$, and $\mathrm{M}$ located on the pump casing, on the bearings housing, and on the AC motor respectively.

The labels of the points P1 to P9 on Figure 1 indicate on which side of the pipe the accelerometers were placed.

The measurements were carried out in four stages.

In the first stage, the throttling valve was closed to prevent the flow and the vibrations associated with flow phenomena. Immediately after starting the pump, vibration levels were measured only at points $P, B$, and $M$ to identify those vibrations caused by the pump, the bearings and the motor when the bearings and the motor are still cold. This stage will be denoted as "cold running".

In the second stage, the throttling valve was kept opened close to its fully opened position, while the butterfly valve was closed progressively from the fully opened position, in which the valve angle $\alpha$ equals $0^{\circ}$, to valve angles of $30^{\circ}, 40^{\circ}$, and $50^{\circ}$. For each valve angle, the pressure drop on the orifice plate mounted on the suction pipe was measured and the vibration levels at the measurement points $\mathrm{P} 1$ to $\mathrm{P} 9$ were recorded. At the points $\mathrm{P}, \mathrm{B}$, and $\mathrm{M}$ vibrations were recorded only for the valve angle $\alpha=0^{\circ}$. Based on the pressure drops on the orifice plate, the flow rates were calculated according to SR EN ISO 5167-2, which is an exact translation in Romanian of ISO 5167-2 [14].

In the third stage, the butterfly valve was kept opened at $\alpha=0^{\circ}$ and the opening of the throttling valve was diminished, so that the discharge decreased below the maximum value attained during the second stage. For this configuration, the vibrations were measured only at points $\mathrm{P}, \mathrm{B}$, and $\mathrm{M}$.

In the fourth stage, the throttling valve was closed completely to prevent the flow and the vibrations were measured again at points $\mathrm{P}, \mathrm{B}$, and $\mathrm{M}$ to identify those vibrations caused by the pump, the bearings and the motor when the bearings and the motor are warm due to the 

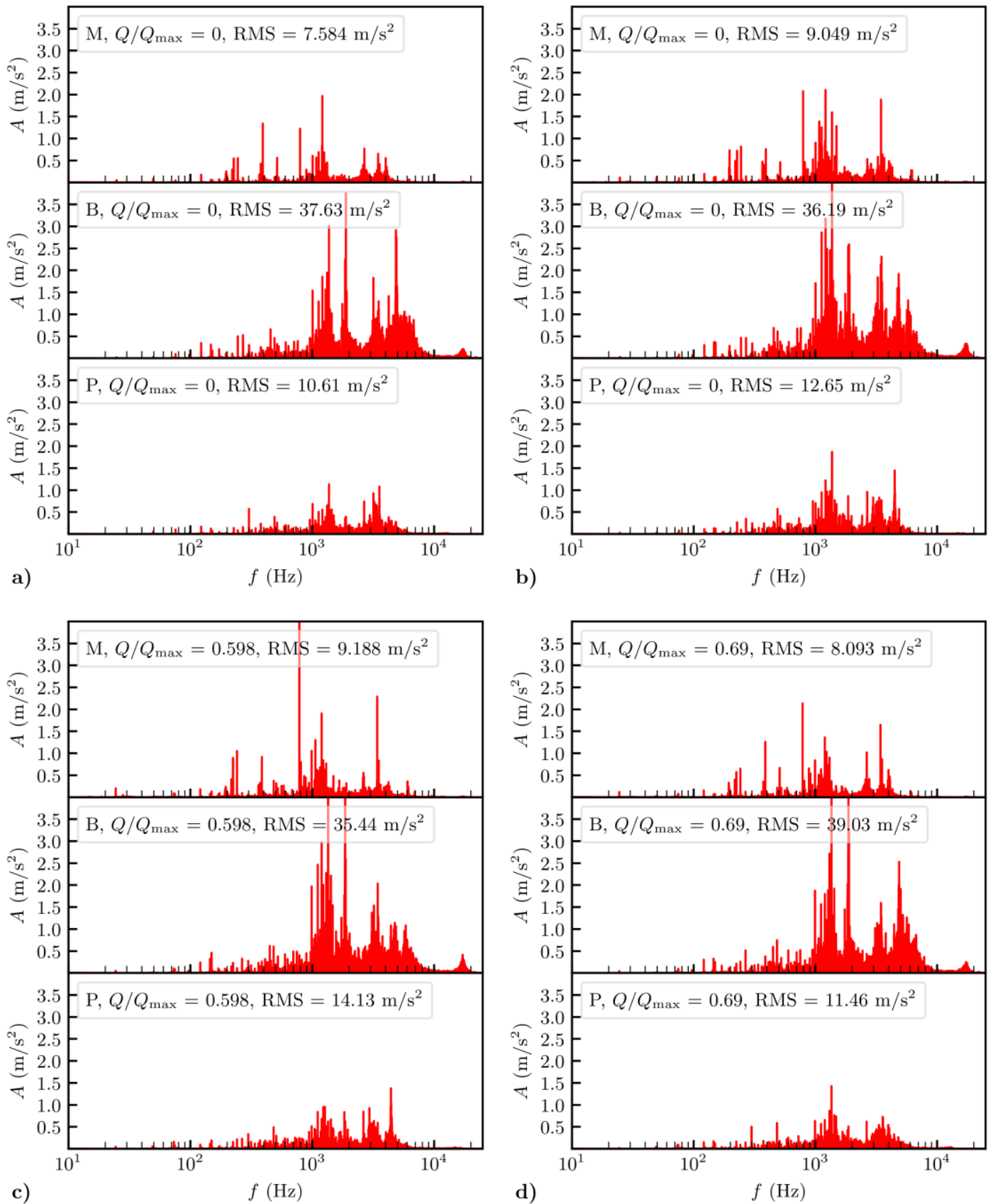

Fig. 2. Frequency spectra of the vibrations measured at different flow rates on the motor, on the bearings housing, and on the pump at a) zero flow rate during cold running, b) zero flow rate during warm running, c) diminished opening of the throttling valve, and d) maximum opening of the throttling valve.

energy dissipated into heat inside the bearings and inside the motor.

The second, third, and fourth stages will be also denoted as "warm running".

\section{Results}

Figure 2 presents frequency spectra obtained by applying the Fast Fourier Transform (FFT) to the vibration signals measured on the pump, on the bearings, and on the motor at different operating regimes. The operating regime is indicated by the relative flow rate calculated as the ratio between the operating flow rate and the maximum flow rate that can be delivered by the pump, $Q / Q_{\max }$. Figures $2 \mathrm{a}$ and $2 \mathrm{~b}$ show results obtained at zero flow rate during cold and warm running respectively. In terms of accelerations, the fundamental frequency of roughly $24.3 \mathrm{~Hz}$ is barely visible, yet not completely absent. The same remark is valid for the blade passing frequency. To better distinguish the fundamental frequency, its harmonics, and other lower frequencies, an integration is required to convert accelerations to velocities or even to displacements. However, the purpose of the study was to investigate not the domain of low frequencies, but that of high frequencies. Therefore, all the results presented in this paper are in terms of accelerations.

The bearings clearly have the highest level of vibrations independently of flow rate and of whether the motor, the bearings, and the pump run cold or warm. Moreover, the signal measured on the bearings housing 
is very noisy in the frequency domain between $350 \mathrm{~Hz}$ and $10 \mathrm{kHz}$. This domain can be split into three subdomains: (i) from $350 \mathrm{~Hz}$ to $800 \mathrm{~Hz}$, (ii) from $800 \mathrm{~Hz}$ to $2.5 \mathrm{kHz}$, and (iii) from $2.5 \mathrm{kHz}$ to $10 \mathrm{kHz}$. In particular, the highest amplitude peaks exceed $3 \mathrm{~m} / \mathrm{s}^{2}$ at frequencies of roughly $1.3 \mathrm{kHz}$ and $2 \mathrm{kHz}$. It is interesting to note that the frequency of $1.3 \mathrm{kHz}$ is roughly ten times the blade passing frequency. Based on previous studies and on manufacturers specifications, it can be assumed that the vibrations in the first frequency subdomain, i.e. below $800 \mathrm{~Hz}$, are caused only by the bearings. An additional source of vibrations is the imbalance of the cantilevered pump impeller caused by the centrifugal force, by the variable and uneven pressure distribution inside the pump volute, and by the hydrodynamic interaction at the pump outlet between the pump impeller and the volute tongue. This imbalance is transmitted through the shaft to the bearings and is likely to be the source of the vibrations having frequencies close to and above $1 \mathrm{kHz}$. However, another in-depth study focused on the pressure pulsations inside the pump volute and at its outlet is required to verify this assumption.

Considering the shape of the humps on the diagrams in Figure 2, it can be stated that the vibrations measured on the motor and on the pump are similar to a large extent to those measured on the bearings housing. The most important difference consists in the significantly lower amplitudes of the vibrations measured on the motor and on the pump. It seems that the vibrations having frequencies higher than $350 \mathrm{~Hz}$ are transmitted from the bearings. The lower amplitudes at the motor could be due to the dampening by the elastic pin bush coupling, while the decrease in amplitudes at the pump could be caused by the flanged couplings with the suction and the pressure pipes, that increase the stiffness of the pump.

A comparison of Figures $2 a$ and $2 b$, i.e. between cold and warm running respectively, suggests that the operating temperature has a certain influence on the vibration levels. On the motor there are visible increases in amplitudes at frequencies above $800 \mathrm{~Hz}$, with a significant increase at roughly $3.5 \mathrm{kHz}$. On the bearings, the amplitudes clearly increase at frequencies around $1.3 \mathrm{kHz}$ and decrease slightly at $5 \mathrm{kHz}$. It is rational to assume that these changes in the vibration levels are caused by the variations of the rotor-stator gap inside the motor and of the clearances inside the bearings due to the increase in the operating temperature.

When Figures $2 \mathrm{a}$ and $2 \mathrm{~b}$ are compared with Figures $2 \mathrm{c}$ and $2 \mathrm{~d}$, it can be observed that there are no significant differences between the vibration levels measured at zero flow rate and those measured when the water is pumped inside the installation. In particular, Figure 2c shows an important increase in the amplitude of the vibrations on the motor at about $800 \mathrm{~Hz}$, but this could have only an electro-magnetic cause related to the change in the load of the motor. Therefore, it can be concluded that the phenomena that take place inside the pump and the bearings are important sources of vibrations having frequencies up to $10 \mathrm{kHz}$.

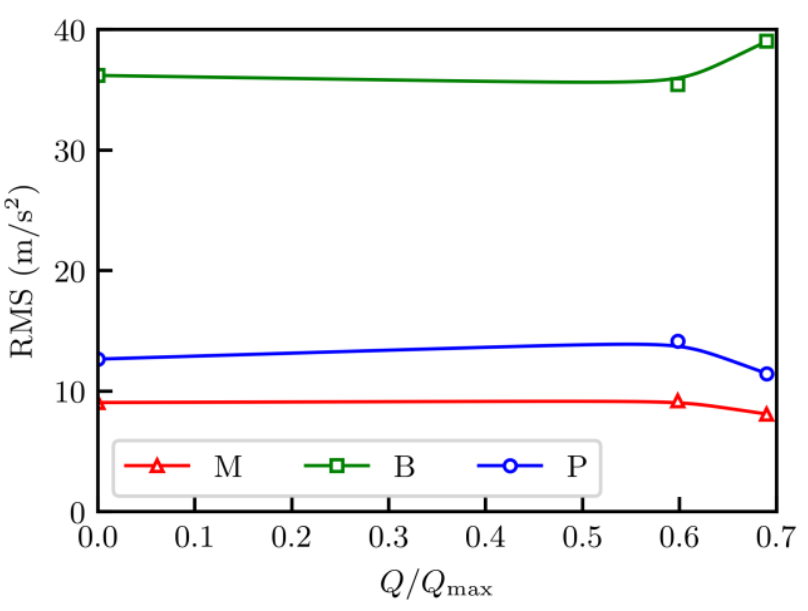

Fig. 3. RMS accelerations of the vibrations measured on the motor, on the bearings housing, and on the pump depending on the relative flow rate.

The RMS accelerations of the vibrations measured on the motor, on the bearings housing, and on the pump depending on the relative flow rate $Q / Q_{\max }$ during warm running, under conditions of non-cavitating flow, are summarized in Figure 3. The motor is the least prone to variations in the RMS acceleration depending on the flow rate. The variation of the RMS acceleration remains low even on the pump and on the bearings. The higher level of vibrations on the bearings in comparison with the pump and the motor is clearly indicated by the significantly higher values of the RMS acceleration. It is interesting to note that on the motor and on the pump the increase in the flow rate leads eventually to a slight decrease in the RMS acceleration, while on the bearings the increase in the flow rate is accompanied by an increase in the RMS acceleration.

Figure 4 presents frequency spectra of the vibrations measured upstream and downstream of the butterfly valve, in points $\mathrm{P} 4$ and $\mathrm{P} 5$ respectively, at different valve angles. It should be mentioned that the noise specific to cavitation was heard after switching the valve flap from $\alpha=30^{\circ}$ to $\alpha=40^{\circ}$. Therefore, the frequency spectra corresponding to the valve angles of $0^{\circ}$ and $30^{\circ}$, presented in Figures $4 \mathrm{a}$ and $4 \mathrm{~b}$ respectively, were obtained in the absence of cavitation, while the frequency spectra corresponding to the valve angles of $40^{\circ}$ and $50^{\circ}$, presented in Figures $4 \mathrm{c}$ and $4 \mathrm{~d}$ respectively, were obtained under conditions of cavitating flow. The blocking mechanism of the butterfly valve did not allow to precisely find the valve angle at which the cavitation is initiated. It should be reminded here that an increase in the valve angle corresponds to a local decrease in the flow area, which favors the increase in the cavitation intensity at the butterfly valve.

As long as the cavitation does not occur (Figs. 4a and $4 \mathrm{~b})$, the frequency spectra of the vibrations measured close to the butterfly valve show larger amplitudes mostly between $100 \mathrm{~Hz}$ and $3 \mathrm{kHz}$. The corresponding vibrations propagate most probably from the pump and from the bearings. In particular, the amplitudes of the vibrations at the blade passing frequency of $121.5 \mathrm{~Hz}$ are visibly higher than those of the vibrations measured on the pump (Fig. 2). This indicates that the vortex 

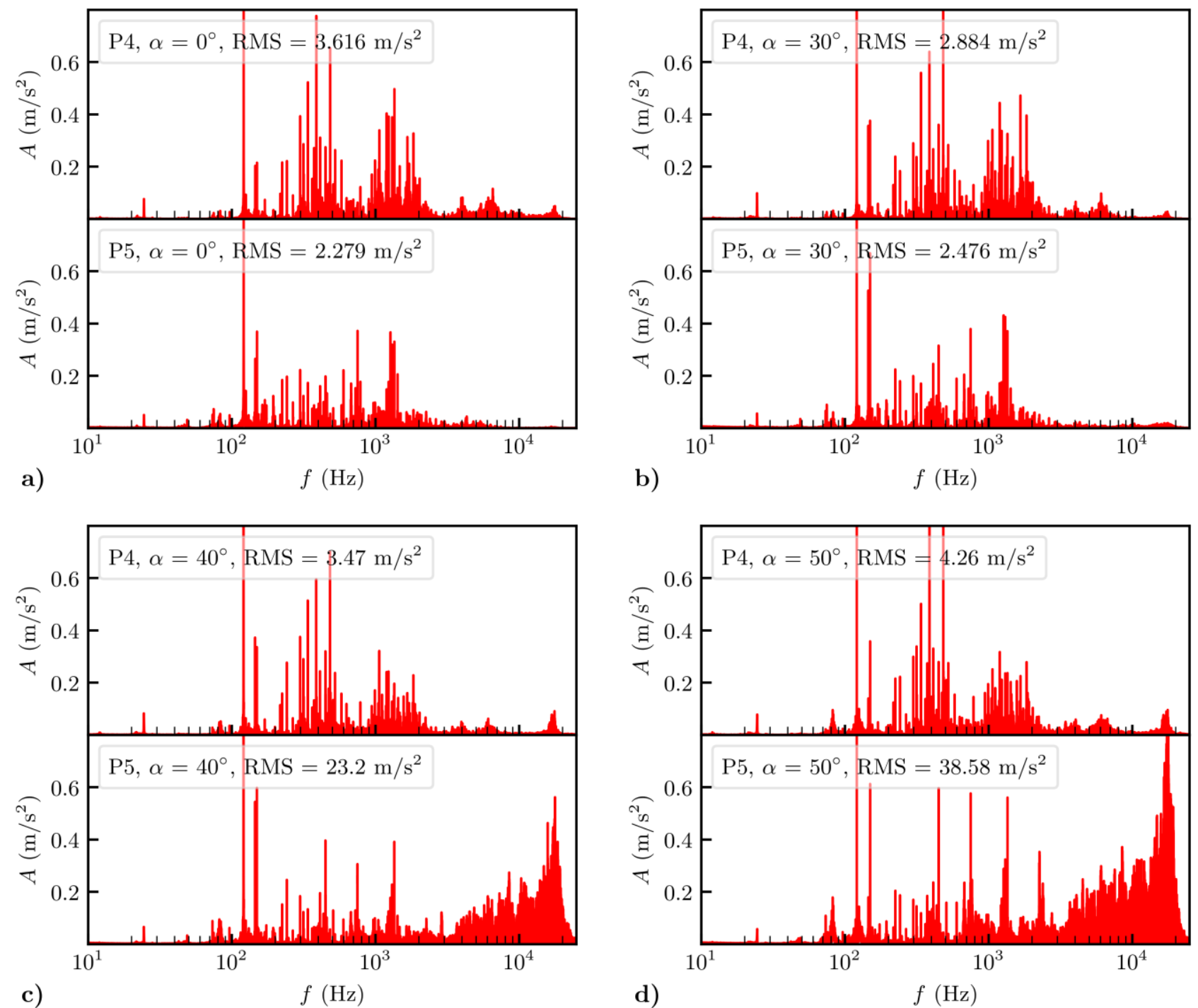

Fig. 4. Frequency spectra of the vibrations measured at points P4 upstream and P5 downstream of the butterfly valve at different valve angles.

shedding at the volute tongue, caused by the hydrodynamic interaction between the blades of the pump impeller and the tongue, produces effects mostly downstream of the pump and to a significantly lesser extent inside the pump.

It is also interesting to note that, although in the absence of cavitation the amplitudes of the vibrations above $3 \mathrm{kHz}$ are very small, they are higher upstream of the butterfly valve, at point $\mathrm{P} 4$, than downstream, at point P5. This suggests that, under conditions of noncavitating flow, the butterfly valve acts as a damper for the high frequency vibrations. The explanation could most probably reside in the increased elasticity of the valve due to its thick rubber lining and to its mounting between flanges using long bolts.

When the cavitation occurs, it has a marginal effect on the vibrations measured upstream of the butterfly valve, at point P4 (Figs. 4c and 4d). As expected, the cavitation has, however, a dramatic effect on the vibrations measured downstream of the valve, at point P5. At frequencies above $3 \mathrm{kHz}$, the cavitation causes vibrations of increasing amplitude as the frequency increases, until a distinct peak is reached at roughly
$18 \mathrm{kHz}$. Beyond this frequency, the amplitudes of the vibrations decrease rapidly. A comparison of Figures $4 \mathrm{c}$ and $4 \mathrm{~d}$ reveals that the amplitudes of the vibrations measured at point P5 and having frequencies above $3 \mathrm{kHz}$ increase also when the cavitation intensity increases due to the increase in the valve angle.

Figure 5 summarizes the RMS accelerations of the vibrations recorded at the measuring points P1 to P9 at different relative flow rates obtained by adjusting the valve angle at the butterfly valve. Before discussing the results, it should be recalled that the decrease in flow rate is due to the progressive closing of the butterfly valve, which diminishes the flow area and, at valve angles greater than $30^{\circ}$, leads to cavitation. From this point of view, the diagrams in Figure 5 should be read from right to left. Downstream of the pump and upstream of the butterfly valve, at points P1, P2, P3, and P4, the decrease in flow rate leads to a decrease in RMS accelerations until the cavitation at the butterfly valve is initiated. The decrease in RMS accelerations is steeper closer to the pump, i.e. at points $\mathrm{P} 1$ and $\mathrm{P} 2$. This decrease could be explained by the decrease in the frequency and the intensity of the vortex shedding at the tongue of the pump 


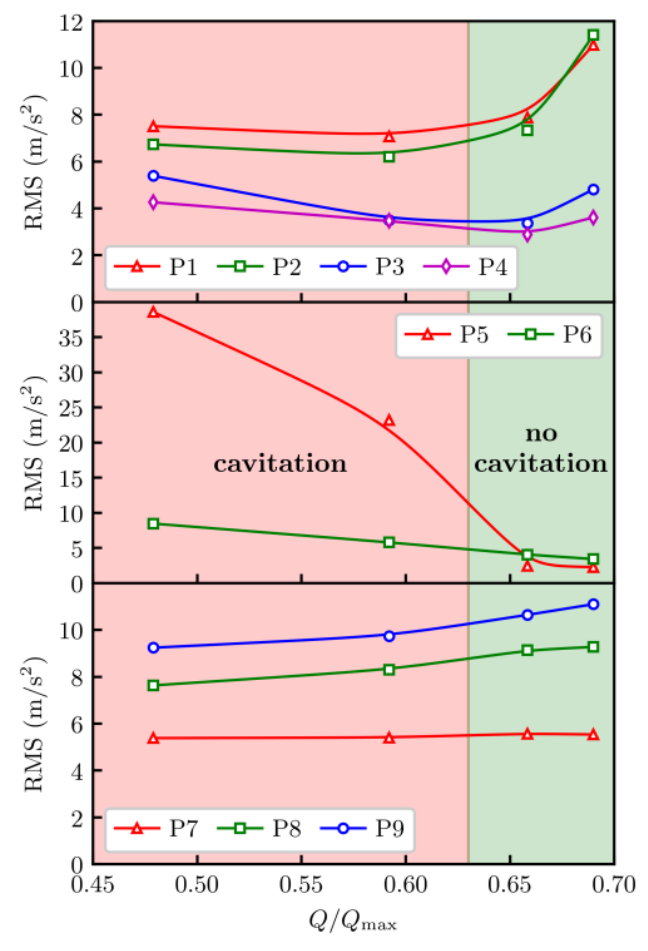

Fig. 5. RMS accelerations of the vibrations recorded at the measuring points $\mathrm{P} 1$ to $\mathrm{P} 9$, depending on the relative flow rate.

volute, at the throttling valve, and at the pipe elbow downstream of the pump as the flow rate decreases. After the cavitation is initiated, the RMS accelerations start to increase as the flow rate continues to decrease and as the cavitation intensity at the butterfly valve increases. Downstream of the butterfly valve, at point P5, the RMS acceleration remains almost constant at a low value of roughly $2 \mathrm{~m} / \mathrm{s}^{2}$ in the absence of cavitation and increases abruptly up to slightly less than $40 \mathrm{~m} / \mathrm{s}^{2}$ as the decrease in flow rate is accompanied by an increase in cavitation intensity. On the water tank, at point P6, the RMS acceleration also increases as the flow rate decreases. Since the water tank is close to the butterfly valve, the increase in RMS acceleration could be related to the increase in cavitation intensity. On the suction pipe, at points $\mathrm{P} 7, \mathrm{P} 8$, and $\mathrm{P} 9$, the decrease in flow rate is accompanied by a decrease in RMS accelerations. The decrease is more pronounced at point $\mathrm{P} 9$, which is the closest to the pump, and negligible at point $\mathrm{P} 7$, which is the furthest from the pump. These last results indicate that the cavitation at the butterfly valve has no influence on the vibration levels on the suction pipe.

\section{Conclusions}

The study presented in this paper aimed to experimentally investigate the vibrations in a water hydraulic system, being particularly focused on those vibrations induced by the occurrence of cavitation at a butterfly valve.

The results obtained suggest that the pump and its bearings are important sources of vibrations with frequencies of up to $10 \mathrm{kHz}$. The increase in flow rate leads to a certain increase in the RMS accelerations of the vibrations measured on the bearings housing.
The occurrence of cavitation downstream of the butterfly valve is accompanied by a significant increase in the amplitudes of the vibrations having frequencies above $3 \mathrm{kHz}$, with a distinct peak at roughly $18 \mathrm{kHz}$. Thus, the results presented indicate that the frequency domain specific to cavitation at a butterfly valve is larger than that presented by Yamada et al. [8]. In terms of global values, the cavitation at the butterfly valve comes with an abrupt increase of about 20 times in the RMS accelerations of the vibrations measured downstream of the valve.

The results obtained could be useful in the management of preventive maintenance of complex water hydraulic systems.

\section{References}

1. A. Ferrari, Proc. R. Soc. A 473: 20160345 (2017), http://dx.doi.org/10.1098/rspa.2016.0345

2. H. Schmidt et al, J. Phys.: Conf. Ser. 656, 012071 (2015), http://iopscience.iop.org/article/10.1088/ 1742-6596/656/1/012071/pdf

3. S.I. Bernad, R.S. Resiga, Modell. Simul. Eng. 2012, 742162 (2012), doi:10.1155/2012/742162

4. J.P. Tullis, J. Hydraulics Div. 99 (1973)

5. S. Moore, Proc. of ACOUSTICS (2016), https:// www.acoustics.asn.au/conference_proceedings/AAS NZ2016/papers/p112.pdf

6. P. Moussou, P. Lafon, S. Potapov, L. Paulhiac, A.S. Tijsseling, RANA, 0404 (2004), https://pure.tue.nl/ ws/portalfiles/portal/2005596

7. G. Baran, F.D. Bunea, G.I. Oprina, Sci. Bull. "Politehnica" Timisoara, 52, 66 (2007)

8. M. Yamada et al., Proc. 27th JSME Tohoku Branch Spring Annual Meeting (1992), https://www.jstage. jst.go.jp/article/tsj1973/26/6/26_6_361/_pdf

9. G. Wang et al., Proc. 9th National Symp. on Cavi. Akiu, Japan (1997)

10. S. Nizuyama, M. Murase, Y. Fuji, Y. Yagi, J. of Env. and Eng., 5, 1 (2010)

11. A. Gribok, S. Patnaik, C. Williams, M. Pattanaik, R. Kanakala, Framework for Structural Online Health Monitoring of Aging and Degradation of Secondary Systems due to some Aspects of Erosion - Technical Report

https://lwrs.inl.gov/Advanced\%20IIC

(2016),

$\% 20$ System\%20Technologies/Framework_Structura 1_Online_Health_Monitoring_of_Aging_Degradatio n.pdf

12. J. Kiesbauer, Hydrocarbon Processing Mag., June (2001), https://www.samson.de/document/ w01040en.pdf

13. J. Kiesbauer, D. Vnucec, M. Roth, B. Stoffel, Hydrocarbon Processing Mag., March (2006), http://www.samson-egeltechniek.nl/6_documentatie/ wa000174en_predicting_cavitation.pdf

14. ISO 5167-2 (2003) 